\title{
Disruption of YPS1 and PEP4 genes reduces proteolytic degradation of secreted HSA/PTH in Pichia pastoris GS115
}

\author{
Min Wu $\cdot$ Qi Shen $\cdot$ Yong Yang $\cdot$ Sheng Zhang $\cdot$ \\ Wen Qu $\cdot$ Jing Chen $\cdot$ Hongying Sun • \\ Shuqing Chen
}

Received: 12 November 2012/ Accepted: 7 March 2013/Published online: 26 March 2013

(C) The Author(s) 2013. This article is published with open access at Springerlink.com

\begin{abstract}
Human serum albumin (HSA) and human parathyroid hormone (1-34) [PTH (1-34)] fusion protein [HSA/PTH (1-34)] is a promising long-acting form of PTH (1-34) for osteoporosis treatment. Secretory expression of intact HSA/PTH (1-34) in Pichia pastoris GS115 was accompanied by two degradation fragments, with molecular weights around $66 \mathrm{kDa}$, in addition to the well-known $\sim 45 \mathrm{kDa}$ HSA-truncated fragment, resulting in a low yield of intact protein. In this study, two internal cleavage sites were identified in the PTH (1-34) portion of the fusion protein by Western Blot analysis. To minimize proteolytic cleavages, several protease genes including PEP4 (encoding proteinase A), PRBI (proteinase B) and seven YPSs genes (yapsin family members) were knocked out respectively by disruption of the individual genes and the selective combinations. Reduced degradation was observed by
\end{abstract}

Electronic supplementary material The online version of this article (doi:10.1007/s10295-013-1264-8) contains supplementary material, which is available to authorized users.

M. Wu · Q. Shen · W. Qu · J. Chen · H. Sun · S. Chen $(\bowtie)$ Institute of Pharmacology and Toxicology and Biochemical Pharmaceutics, College of Pharmaceutical Sciences, Zhejiang University, 866 Yuhangtang Road, Hangzhou 310058, Zhejiang, People's Republic of China

e-mail: chenshuqing@zju.edu.cn

Y. Yang

Robert W. Holley Center for Agriculture and Health,

USDA-ARS, Ithaca, NY 14853, USA

\section{S. Zhang}

Institute of Biotechnology and Life Sciences Biotechnologies, Cornell University, Ithaca, NY 14853, USA

J. Chen

School of Pharmaceutical Sciences, University of Shizuoka,

Shizuoka 4228526, Japan single disruption of either PEP4 gene or YPS1 gene, and the lowest level of degradation was observed in a pep $4 \triangle$ yps $1 \triangle$ double disruptant. After $72 \mathrm{~h}$ of induction, more than $80 \%$ of the HSA/PTH (1-34) secreted by the pep $4 \triangle y p s 1 \triangle$ double disruptant remained intact, in comparison to only $30 \%$ with the wild-type strain.

Keywords Heterologous protein expression · Proteolytic degradation - Pichia pastoris - Yapsin - Proteinase A

\section{Introduction}

Human parathyroid hormone (1-34) [PTH (1-34)] covers most of the hormonal actions of intact human parathyroid hormone [PTH (1-84)], namely regulating calcium/phosphate homeostasis and controlling bone turnover in vertebrates by activating specific receptors located on osteoblastic and renal tubular cell [16]. PTH (1-34) has now been developed as a promising agent in the treatment of osteoporosis $[17,25]$. However, the short half-life of unmodified PTH (1-34) at $\sim 1 \mathrm{~h}$ in humans makes frequent injection (once daily) necessary in long-term dosing regimens (1-2 years), and therefore limits its clinical applications.

Albumin fusion technology is a strategy for decreasing clearance of short-acting drugs such as PTH (1-34), providing the advantageous pharmacokinetic properties of human serum albumin (HSA) due to its long biological half-life $\left(t_{1 / 2}\right.$ of 19 days) to its fusion partner [22]. Previously, in our efforts to extend the biological half-life of native PTH (1-34), we have constructed an HSA/PTH (1-34) fusion protein via fusion of $\mathrm{N}$-terminus of PTH (1-34) to C-terminus of HSA, and could obtain the recombinant biologically active fusion protein using Pichia pastoris expression system [4]. Unfortunately, when HSA/PTH (1-34) was expressed in Pichia pastoris 
strain GS115, two degradation fragments of around $66 \mathrm{kDa}$ were found, in addition to a $\sim 45 \mathrm{kDa}$ HSA-truncated fragment. The formation of a $\sim 45 \mathrm{kDa}$ fragment is well-known from secreted production of HSA alone, in both Saccharomyces cerevisiae [13] and P. pastoris [14]. The inhomogeneous expression of HSA/PTH (1-34) fusion protein made it more difficult and more time-consuming for downstream purification of intact recombinant protein with high purity.

It has become increasingly clear that proteolytic degradation of the recombinant gene products by host-specific proteases is one of the major problems hindering effective production and purification of heterologous proteins from yeasts [21, 27]. And genetic manipulation of host strain by systematic disruption analysis of the key proteases seems to be an effective solution to decrease proteolytic degradation $[5,6,9,10,15]$.

Yapsins are a family of glycosylphosphatidylinositol (GPI)linked aspartyl proteases having specificity to cleave at the C-terminal side of basic amino acids [2]. A number of studies have reported the involvement of yapsins, particularly yapsin 1 , in the cleavage of various heterologous proteins produced in yeast $[1,12,13]$. It was reported that the $\sim 45 \mathrm{kDa} H S A-$ truncated fragment produced in $S$. cerevisiae [13] was attributable to yapsin 1, and a partial reduction of the similar $\sim 45 \mathrm{kDa}$ HSA-truncated fragment in $P$. pastoris [26] was also found by YPS1 disruption. PTH (1-84) was also susceptible to yapsins when expressed in $S$. cerevisiae and the use of multiple-yapsin-deficient mutant was efficient in preventing the proteolytic degradation [5]. YPS1 gene of P. pastoris was first cloned and characterised by Werten and Wolf [24], and the authors found that the yps 1 -disrupted $P$. pastoris strain was beneficial for secreting production of collagen-inspired gelforming polymers [20]. Besides, Yao et al. [26] found that the significant reduction of HSA-AK15 (R13 K) degradation, which occurred in the sequence of AK15 (R13 K), was achieved by the YPS1 disruption in P. pastoris strain.

Given this background, we hypothesized that degradation of HSA/PTH (1-34) in P. pastoris may be due to the yapsin family. The $9.43 \mathrm{Mbp}$ genomic sequence of $P$. pastoris strain GS115 in 2009 revealed the presence of other six putative yapsin genes (YPS2, YPS3, YPS7, MKC7, YPS', YPS ${ }^{\prime \prime}$ ) [7]. Five of these were disrupted and their effects on cell-wall integrity were investigated [8]. To the best of our knowledge, the present work is the first to study the effect of the individual disruption of seven putative yapsins on the degradation of recombinant proteins in $P$. pastoris. In this study, firstly, the inhomogeneous products of HSA/PTH (1-34) were characterized by biochemical and Western blot analyses. Secondly, in order to investigate the proteolytic effect of each yapsin member, seven single-yapsin-deficient GS115 mutants were constructed. Two prominent vacuolar proteases (proteinase A and proteinase B) were also evaluated, as vacuolar proteases are often responsible for a large fraction of total cellular proteolysis [21]. As a result, we have successfully identified that both PEP4 disruption and YPS1 disruption were beneficial for degradation reduction of HSA/PTH (1-34) fusion protein via visualized PAGE analysis. Thirdly, to achieve efficient production of intact HSA/PTH (1-34), we constructed a double gene disruptant (proteinase A and yapsin 1 double disrupted) as an effective host strain. The double disruptant was advantageous over the wild-type strain both in shake-flask and in bioreactor fermentation, which would allow high yield of this interesting protein and thereby simplify purification processes in industrial applications.

\section{Materials and methods}

Strains and media

The $P$. pastoris strains used in this study are listed in Table 1. Strains were cultured in the following media: YPD (1\% yeast extract, $2 \%$ peptone, $2 \%$ glucose) for subcultivation; BMGY (1\% yeast extract, $2 \%$ peptone, $1.34 \%$ YNB, $4 \times 10^{-5} \%$ biotin, $1 \%$ glycerol, $100 \mathrm{mM}$ potassium phosphate $\mathrm{pH}$ 6.0) and BMMY (same as BMGY substituting $1 \%$ glycerol with $1 \%$ methanol) for recombinant protein production. YPD Zeocin ${ }^{+}$plates (YPD plus $2 \%$ agar and $50 \mu \mathrm{g} / \mathrm{mL}$ Zeocin) were used for screening of $Y P S$-deficient strains. Yeast competent cells transformed with HSA/PTH (1-34) expression vector were plated onto RDB His ${ }^{-}$plates (1 M sorbitol, $2 \%$ glucose, $1.34 \% \mathrm{YNB}$, $4 \times 10^{-5} \%$ biotin, $0.005 \%$ amino acids, $2 \%$ agar).

\section{Construction of HSA/PTH (1-34) expression vector}

For creating the HSA/PTH (1-34) fusion protein [Genbank accession no. JN711437], the C-terminus of HSA and the N-terminus of PTH (1-34) were genetically linked by a flexible linker GlyGlyGlyGlySer, as previously reported [4]. In this study, to secrete the fusion protein with its native $\mathrm{N}$-terminus, the $K E X 2$ cleavage site was positioned precisely in front of the first aa of HSA/PTH (1-34) protein sequence. pPIC9 was chosen as the expression vector. The detailed method for plasmid construction is described in supplementary materials and methods.

Construction of protease-deficient strains

GS115 pep4 $\triangle$ and GS115 pep $4 \triangle p r b 1 \triangle$, also called SMD1168 and SMD1163, are commercially available (from Invitrogen, see Table 1). The seven single-yapsindeficient GS115 mutants (GS115 yps1 $\triangle$, GS115 yps $2 \triangle$, GS115 yps $3 \triangle$, GS115 yps7 $\triangle$, GS115 mkc7 $\triangle$, GS115 yps $\triangle$, GS115 yps" $\triangle$ ) were constructed by deleting the full ORF sequence of each yapsin gene, as shown in Fig. 1. The 
Table 1 Pichia pastoris strains used in this study

\begin{tabular}{|c|c|c|c|c|c|}
\hline Strain name & $\begin{array}{l}\text { Disrupted } \\
\text { genes }\end{array}$ & Genbank accession no. & Protease family & Genotype & Reference \\
\hline WT GS115 & - & - & - & his4 & $\begin{array}{l}\text { Purchased from } \\
\text { Invitrogen }\end{array}$ \\
\hline GS115 yps1 $\triangle$ & YPS1 & XM_ 002493977 & Aspartic-type endopeptidase & his4 yps1::Sh ble & This study \\
\hline GS115 yps $2 \triangle$ & YPS2 & XM_002492474 & Aspartic-type endopeptidase & his4 yps2::Sh ble & This study \\
\hline GS115 yps $3 \triangle$ & $Y P S 3$ & XM_002492478 & Aspartic-type endopeptidase & his4 yps3::Sh ble & This study \\
\hline GS115 yps7 $\triangle$ & YPS7 & XM_002942575 & Aspartic-type endopeptidase & his4 yps7::Sh ble & This study \\
\hline GS115 mkc7 $\triangle$ & $M K C 7$ & XM_002489993 & Aspartic-type endopeptidase & His4 mkc7::Sh ble & This study \\
\hline GS115 yps $\triangle$ & $Y P S^{\prime a}$ & XM_002492327 & Aspartic-type endopeptidase & his4 yps'::Sh ble & This study \\
\hline GS115 yps" $\triangle$ & $Y P S^{\prime \prime}$ & XM_002493054 & Aspartic-type endopeptidase & his4 yps"::Sh ble & This study \\
\hline GS115 pep4 $\triangle$ & PEP4 & XM_002493288 & Aspartic-type endopeptidase & his4 pep4::URA3 & $\begin{array}{l}\text { Purchased from } \\
\text { Invitrogen }\end{array}$ \\
\hline $\begin{array}{l}\text { GS115 } \\
\text { pep4prb1 } \triangle\end{array}$ & $\begin{array}{l}\text { PEP4, } \\
\text { PRB1 }\end{array}$ & $\begin{array}{l}\text { XM_002493288, } \\
\text { XM_002489831 }\end{array}$ & $\begin{array}{l}\text { Aspartic-type endopeptidase, serine-type } \\
\text { endopeptidase }\end{array}$ & $\begin{array}{l}\text { his4 pep } 4:: U R A 3 \\
\text { prb1::URA3 }\end{array}$ & $\begin{array}{l}\text { Purchased from } \\
\text { Invitrogen }\end{array}$ \\
\hline $\begin{array}{l}\mathrm{GS} 115 \\
\text { pep4 } \triangle \text { yps } 1 \triangle\end{array}$ & $\begin{array}{l}\text { PEP4, } \\
\text { YPS1 }\end{array}$ & $\begin{array}{l}\text { XM_002493288, } \\
\text { XM_002493977 }\end{array}$ & $\begin{array}{l}\text { Aspartic-type endopeptidase, aspartic- } \\
\text { type endopeptidase }\end{array}$ & $\begin{array}{l}\text { his4 pep } 4:: U R A 3 \\
\text { yps } 1:: \text { Sh ble }\end{array}$ & This study \\
\hline
\end{tabular}

${ }^{a} Y P S^{\prime}$ and $Y P S^{\prime \prime}$ represent additional putative yapsin-like genes in the $P$. pastoris genome, not unequivocally attributable to previously reported yapsin homologs

a

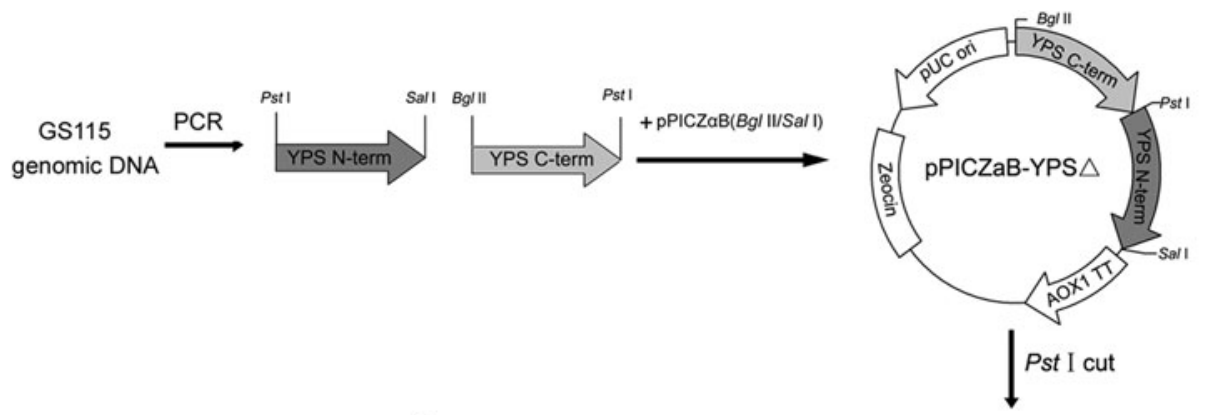

C

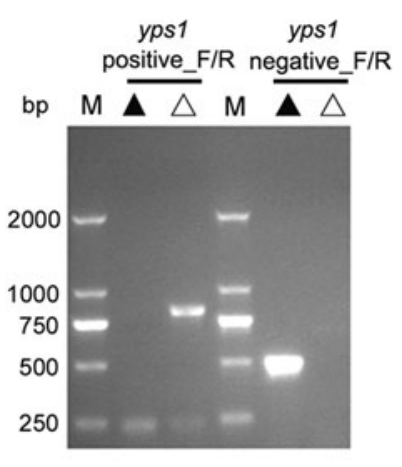

b

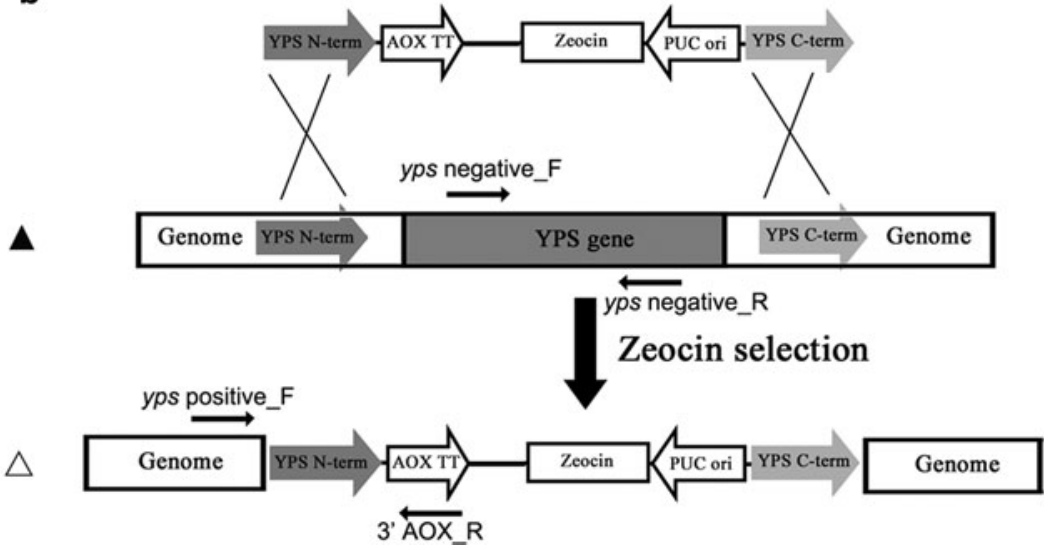

Fig. 1 Schematic representation of experimental procedures for $Y P S$ gene disruption and confirmation. a Construction strategy for $Y P S$ gene disruption cassette. b Disruption of YPS gene by homologous recombination. The symbols filled triangle and open triangle indicate parental strain and the yps disruptant, respectively. Primer pair yps

construction of GS115 pep4 $\triangle$ yps $1 \triangle$ mutant was carried out in a similar manner from GS115 pep $4 \triangle$. Briefly, for each YPS gene, a 200-300 bp DNA fragment containing
positive_F/ $3^{\prime} A O X \_R$ was used to confirm the integration of the gene disruption cassette and primer pair yps negative_F/yps negative_R was used to confirm the deletion of $Y P S$ gene. $\mathbf{c}$ PCR results of correct yps $1 \triangle$ transformant as an example

the $5^{\prime}$ homology arm of the YPS gene was amplified from GS115 genomic DNA using primers YPS_NF and YPS_NR. Another 200-300 bp DNA fragment containing 
the $3^{\prime}$ homology arm of the $Y P S$ gene was amplified using primers YPS_CF and YPS_CR. The two homology arms above were identified via DNA sequencing and sequentially subcloned into a $\sim 2.2 \mathrm{~kb} \mathrm{Bgl} \mathrm{II/Sal} \mathrm{I} \mathrm{plasmid} \mathrm{frag-}$ ment from pPICZ $\alpha \mathrm{B}$, containing a Sh ble gene (zeocin resistance cassette), to give the new plasmid pPICZ $\alpha \mathrm{B}-$ YPS $\triangle$. The resultant vector was then linearized with Pst I and introduced into competent cells of GS115 by electroporation to stimulating the homologous recombination at the corresponding YPS locus of the P. pastoris genome. Transformed cells were poured on YPD Zeocin ${ }^{+}$plates and incubated at $30{ }^{\circ} \mathrm{C}$ for 3-4 days. PCR analysis was used to screen Zeocin ${ }^{+}$transformants. The correct yps disruptant would give a specific PCR fragment with primer pair yps positive_F/ $3^{\prime} A O X \_R$ and show no specific fragment with primer pair yps negative_F/yps negative_R. All primers used for construction of pPICZ $\alpha \mathrm{B}-Y P S \triangle$ and detection of yps-deficient strain are listed in Table S1.

Transformation and screening of fusion protein expressing strains in shake flask

All $P$. pastoris strains were transformed by electroporation with Sal I-cut fragment of pPIC9-HSA/PTH (1-34), and plated on RDB His ${ }^{-}$plates. For each strain, 3-6 clones were randomly selected and verified for integration of HSA/PTH (1-34) fusion gene by PCR. Three batches of expression were done. For each batch, seeds of all strains were grown at $30{ }^{\circ} \mathrm{C}$ to an comparative $\mathrm{OD}_{600}$ $\left(\mathrm{OD}_{600}=2-4\right.$ for batch $1, \mathrm{OD}_{600}=3-4$ for batch 2 , $\mathrm{OD}_{600}=2-3$ for batch 3) in $20 \mathrm{ml}$ BMGY before methanol induction. The cells were harvested by centrifugation at $1,500 \mathrm{~g}$ for $10 \mathrm{~min}$, resuspended in $20 \mathrm{ml}$ BMMY medium and incubated at $30{ }^{\circ} \mathrm{C}$ with constant shaking. Methanol (100\%) was added to a final concentration of $1 \%$ every $24 \mathrm{~h}$ to maintain induction up to $96 \mathrm{~h}$. The culture supernatant was analyzed by reducing SDS- and native PAGE.

HSA/PTH (1-34) expression by fed-batch fermentation

For pre-culture, $50 \mathrm{ml}$ YPD were inoculated with $0.5 \mathrm{ml}$ frozen glycerol stock of GS115 pep4 $\triangle$ yps $\triangle \triangle$ (or wild-type GS115) and incubated $24 \mathrm{~h}$ at $30{ }^{\circ} \mathrm{C}$ and $150 \mathrm{rpm}$. This first pre-culture was inoculated into $600 \mathrm{ml}$ BMGY for $20-24 \mathrm{~h}$ at $30{ }^{\circ} \mathrm{C}$ and $150 \mathrm{rpm}$ and grown to $\mathrm{OD}_{600}=2-6$. This second pre-culture was used as inoculum.

The fermentation was carried out in a $30 \mathrm{~L}$ bioreactor (GUJS-10-30C, Orient Bioengineering Equipment and Technology Co. Ltd, China) with a working volume of $12 \mathrm{~L}$ BSM (26.7 ml/L phosphoric acid, $0.93 \mathrm{~g} / \mathrm{L}$ calcium sulfate, $18.2 \mathrm{~g} / \mathrm{L}$ potassium sulfate, $14.9 \mathrm{~g} / \mathrm{L}$ magnesium sulfate-
$7 \mathrm{H}_{2} \mathrm{O}, 4.13 \mathrm{~g} / \mathrm{L}$ potassium hydroxide, $40 \mathrm{~g} / \mathrm{L}$ glycerol) supplemented with $4.35 \mathrm{ml} / \mathrm{L} \mathrm{PTM}_{1}$ trace salts $(6.0 \mathrm{~g} / \mathrm{L}$ cupric sulfate $-5 \mathrm{H}_{2} \mathrm{O}, \quad 0.08 \mathrm{~g} / \mathrm{L}$ sodium iodide, $3.0 \mathrm{~g} / \mathrm{L}$ manganese sulfate $-\mathrm{H}_{2} \mathrm{O}, 0.2 \mathrm{~g} / \mathrm{L}$ sodium molybdate $-2 \mathrm{H}_{2} \mathrm{O}$, $0.02 \mathrm{~g} / \mathrm{L}$ boric acid, $0.5 \mathrm{~g}$ cobalt chloride, $20.0 \mathrm{~g} / \mathrm{L}$ zinc chloride, $65.0 \mathrm{~g} / \mathrm{L}$ ferrous sulfate $-7 \mathrm{H}_{2} \mathrm{O}, 0.2 \mathrm{~g} / \mathrm{L}$ biotin, $5.0 \mathrm{ml}$ sulfuric acid). The temperature was maintained at $28{ }^{\circ} \mathrm{C}$ and the $\mathrm{pH}$ at 5.5 (controlled with ammonium hydroxide). The dissolved oxygen (DO) was set above $20 \%$. A 6-hour glycerol fed-batch was performed at a feed rate of $217.8 \mathrm{ml}$ glycerol/h (containing $12 \mathrm{ml} / \mathrm{L} \mathrm{PTM}_{1}$ trace salts) to increase cell mass. After the glycerol fedbatch, cells were hungered for $2 \mathrm{~h}$ to fully adapt to methanol, which was confirmed by a sharp decrease in DO. During the methanol fed-batch, methanol supplemented with $12 \mathrm{ml} / \mathrm{L} \mathrm{PTM}_{1}$ and $2 \%$ casamino acids was used and the final concentration of methanol was maintained at $0.5 \%$ by controlling the feeding rate.

Gel electrophoresis (PAGE) and western blot analysis

Culture supernatant was obtained after centrifuging $1 \mathrm{ml}$ of culture broth. For reducing SDS-PAGE analysis, the culture supernatant was mixed with an appropriate volume of $5 \times$ reducing-PAGE loading buffer containing $60 \mathrm{mM}$ Tris-HCl (pH 6.8), $0.1 \%$ (W/V) bromophenol blue, $25 \%$ (V/V) glycerol, $2 \%(\mathrm{~W} / \mathrm{V})$ SDS and $14.4 \mathrm{mM} \beta$-mercaptoethanol. The mixed samples were then boiled for $5 \mathrm{~min}$ and loaded on a $8 \%$ SDS/polyacrylamide gel. For native PAGE analysis, the culture supernatant was mixed with an appropriate volume of $5 \times$ native-PAGE loading buffer containing $312.5 \mathrm{mM}$ Tris- $\mathrm{HCl}(\mathrm{pH} 6.8), 0.1 \%$ (W/V) bromophenol blue and $25 \%(\mathrm{~V} / \mathrm{V})$ glycerol. The mixed samples were directly loaded on a $8 \%$ polyacrylamide gel. The gels were stained with Coomassie brilliant Blue R-250.

For Western blotting, proteins separated by native PAGE gel were electrophoretically transferred to a PVDF membrane. Primary antibodies were rabbit anti-PTH (1-17) polyclonal antibody, guinea pig anti-PTH (17-34) polyclonal antibody and rabbit anti-HSA polyclonal antibody (seek Supplementary Materials and Methods for the generation of the above three antibodies.) in three separate experiments. Secondary antibodies were HRP conjugated IgG from goat against rabbit and guinea pig, respectively. Immunoreactions were detected using 3,3'-diaminobenzidine (DAB).

Protein relative quantization in PAGE gel

The proportion of intact ( $i$ fragment)and degraded HSA/PTH (1-34) ( $d 1$ fragment and $d 2$ fragment) in the total protein was determined by scanning and analyzing the Coomassie bluestained PAGE gel with laser densitometry (Bio-Rad 
Universal Hood II scanner) and Quantity One 1-D Analysis Software version 4.5.0 (Bio-Rad). The band densities were calculated by trace-tracking and Gauss-modeling calibration according to manufacturer's instruction.

\section{Molecular weight assay of intact HSA/PTH (1-34)}

The $i$ fragment was obtained by a two-step chromatography purification (Phenyl Sepharose FF, Q Sepharose XL, GE Healthcare) for MALDI-TOF mass spectrometry. Native PAGE analysis of the final purified sample is provided as Fig. S3. Sample was mixed with Sinapic Acid $(10 \mu \mathrm{g} / \mathrm{ul}$ dissolved in $30 \%$ acetonitrile and $0.3 \%$ TFA), spotted on a target plate and analyzed on a $5800 \mathrm{MALDI-TOF/TOF}$ analyzer (AB Sciex). Linear mode with $20 \mathrm{kV}$ acceleration voltage was used, with BSA as internal calibration. For the MS spectrum, 1,000 laser shots were accumulated.

\section{Results}

PAGE analysis of secreted HSA/PTH (1-34) from GS115

The GS115 strain harboring one copy of HSA/PTH (1-34) gene was methanol-induced for $96 \mathrm{~h}$ by ordinary shakeflask. The culture supernatants at different induction times were loaded on reducing SDS-PAGE or native PAGE. Figure 2 shows that in addition to $\sim 45 \mathrm{kDa}$ HSA-truncated fragment, there were three bands on native PAGE (Fig. 2b, band $i, d l, d 2)$ and they appeared as a broad band around $66 \mathrm{kDa}$ on reducing SDS-PAGE (Fig. 2a). To test the stability of expressed HSA/PTH (1-34) in the GS115 supernatants, the 3-day induced supernatant was subsequently filtrated using a $0.22 \mu \mathrm{m}$ filter membrane. The cell-free supernatant was then incubated for up to $48 \mathrm{~h}$ at $37{ }^{\circ} \mathrm{C}$. Bands $i$ and $d l$ disappeared gradually after $48 \mathrm{~h}$ incubation and were eventually converted to band $d 2$ (Fig. 3, lane 4 ). In addition, the incubation time course ( $24 \mathrm{~h}$ vs. $48 \mathrm{~h}$ ) showed that band $i$ was less stable than $d l$. But when the supernatant was incubated at $60{ }^{\circ} \mathrm{C}$ for $1 \mathrm{~h}$ prior to $37^{\circ} \mathrm{C}$ incubation, bands $i$ and $d l$ appeared to be relatively stable (Fig. 3, lane 2). Since pre-incubation of the supernatant at higher temperature such as $60{ }^{\circ} \mathrm{C}$ helps to reduce protease activities in the culture supernatant [3], our observations suggest that the heterogeneity of HSA/PTH (1-34) fusion protein during induction is probably caused by proteolytic degradation.

Western blot analysis

Before Western blot analysis, N-terminal sequence of fragments $i$ and $d 2$ were performed. Both of them showed identical sequence of DAHKS..., which matches exactly the N-terminus of mature HSA, indicating correct $\mathrm{N}$-terminal process.

Two PTH antibodies [anti-PTH (1-17) and anti-PTH (1734)] were utilized for further identification of the integrity of PTH (1-34) portion of fusion protein and its degraded products. As expected, Western blot analysis showed that all the three bands $(i, d l$ and $d 2)$ reacted equally well with HSA antibody (Fig. 4a, b). However, as shown in Fig. 4c, d, band $d 2$ reacted neither with PTH (1-17) antibody nor with PTH (17-34) antibody. Band $i$ reacted with both antibodies of PTH as did band $d l$. Moreover, band $d l$ showed equal affinity to PTH (1-17) antibody, but lower affinity to the PTH (17-34) as compared to band $i$ (Fig $4 \mathrm{~b}, \mathrm{c}, \mathrm{d}$ ). The band density ratios of $i / d l$ in the immunoreaction with PTH (1-17) antibody and HSA antibody were both around 0.4, whereas in the immunoreaction with PTH (17-34) antibody, the density ratio of $i / d l$ was about 2, as plotted in Fig. 4e. From the combined results above we speculate that band $i$ consists of intact HSA/ PTH (1-34), while band $d l$ and $d 2$ are degradation fragments from the cleavage at the $\mathrm{C}$-terminal region of fusion protein. The cleavage sites of HSA/PTH (1-34) resulting in degradation fragments $d 1$ and $d 2$ are supposedly within the PTH (17-34) and the PTH (1-17) portions, respectively, as illustrated in Fig. 5.

\section{Mass spectrometry of intact HSA/PTH (1-34) fusion} protein

To further confirm that the $i$ fragment consists of intact HSA/PTH (1-34), the purified $i$ fragment was analyzed by MALDI-TOF mass spectrometry. As shown in Fig. 6. The $i$ fragment showed a molecular weight of 70,887.2656 Da, which is very close to the theoretical molecular mass deduced from its amino acid sequence (70,887.49 Da).
Fig. 2 Time-course analysis of secreted HSA/PTH (1-34) in GS115. a reducing SDSPAGE.b native PAGE. Lane $M$ molecular weight marker; lane $1-4$ culture supernatants after 24, 48, 72, $96 \mathrm{~h}$ methanol induction

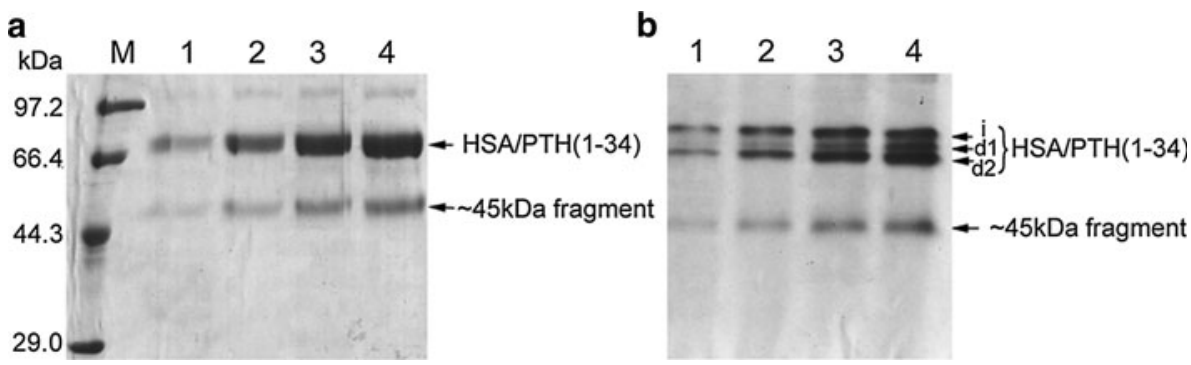




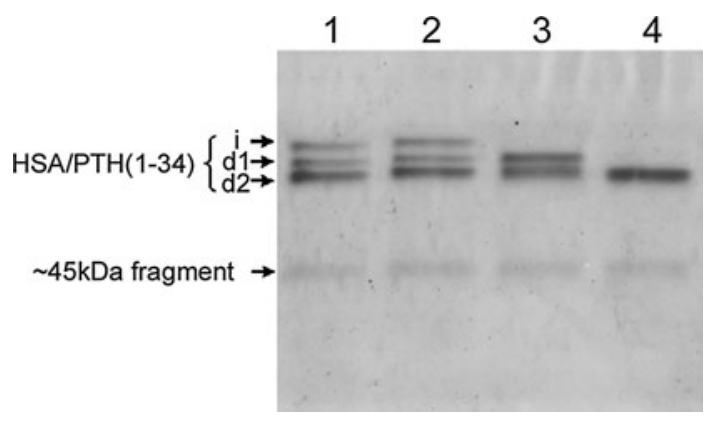

Fig. 3 Stability of HSA/PTH (1-34) inductive supernatant incubated at $37^{\circ} \mathrm{C}$ for different times by native PAGE analysis. Lane $137^{\circ} \mathrm{C}$ $0 \mathrm{~h}$; lane $260{ }^{\circ} \mathrm{C} 1 \mathrm{~h}$ prior to $37^{\circ} \mathrm{C} 24 \mathrm{~h}$; lane $337^{\circ} \mathrm{C} 24 \mathrm{~h}$; lane 4 $37{ }^{\circ} \mathrm{C} 48 \mathrm{~h}$

Effect of different protease-deficient strains on proteolysis by shake flask cultivation

To explore the effect of each yapsin member in proteolysis of HSA/PTH (1-34), seven single-yapsin-deficient GS115 mutants were constructed and tested. For each strain, a clone containing one copy of integrated HSA/PTH (1-34) gene was selected for the comparable cultivation (verified by quantitative real time-PCR, data not shown). Three batches of culture supernatants were obtained for densitometry and statistical analysis after $72 \mathrm{~h}$ induction. As shown in Fig. 7 (lane 4-11), among the seven single-yapsin-deficient strains, the proportion of intact HSA/PTH (1-34) was increased significantly only in yps $1 \triangle$ disruptant, in which the degradation fragment $\mathrm{d} 2$ was considerably diminished but not completely disappeared, while no visualized change was observed for $d l$ fragment. For the other six single-yapsin-deficient strains, the band patterns of each disruptant were quite similar with wild-type strain when visualized from PAGE gels. For the seven singleyapsin-deficient strains, culture supernatants after $24 \mathrm{~h}$ induction were also analyzed, and the results were similar to $72 \mathrm{~h}$ induction, that is the yps $1 \triangle$ disruptant displayed a visible reduction of fragment $d 2$ while the effects of the other six single-yapsin-deficient strains were not significant (see Fig. S1). These observations suggest that yapsin 1 played a role in proteolysis of HSA/PTH (1-34), particularly in generation of fragment $d 2$.

Proteinase A and proteinase B are two major vacuolar proteases which also regulate activities of many other
Fig. 4 Western blot analysis of HSA/PTH (1-34) products. a native PAGE analysis; b immunoreaction with HSA antibody; c immunoreaction with PTH (1-17) antibody; d immunoreaction with PTH (17-34) antibody. Lane $M$ prestained molecular weight marker; lane 1 inductive supernatant from strain GS115 transformed with pPIC9-HSA/ PTH (1-34); lane 2 inductive supernatant from strain GS115 transformed with pPIC9 as a negative control. e Relative band density ratio of $i / d l$. Band densities are quantified by densitometric analyses with Quantity One Software version 4.5.0 (Bio-Rad). Data are mean \pm S.D from three independent experiments

Fig. 5 Schematic representation of cleavage sites in HSA/PTH (1-34). Number 17 refers to the aa positions in the PTH (1-34) sequence
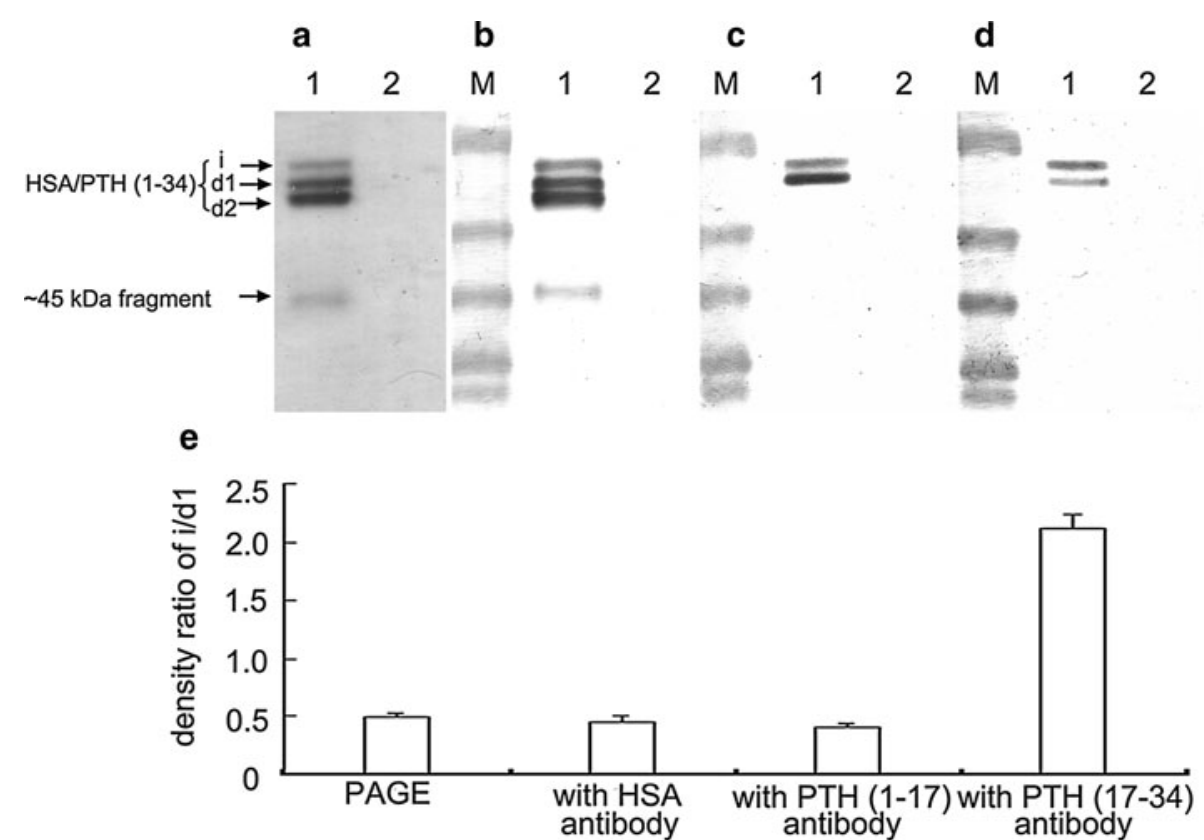

17

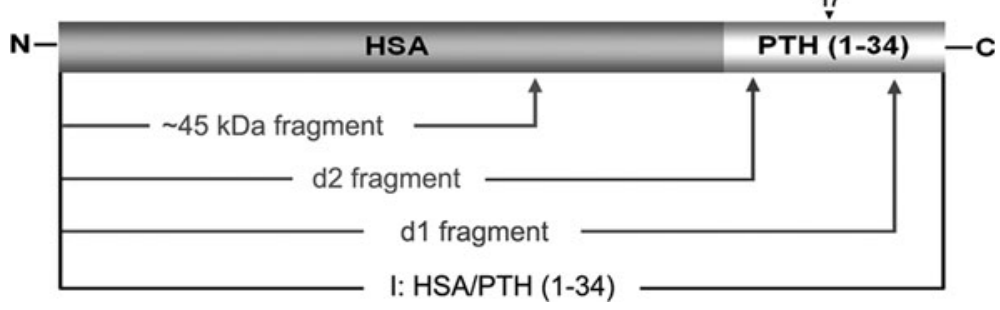


Fig. 6 Molecular weight determination of HSA/PTH (1-34) by MALDI-TOF mass spectrometry. Peak 1 represents the BSA internal standard, peak 2 represents HSA/PTH (1-34)

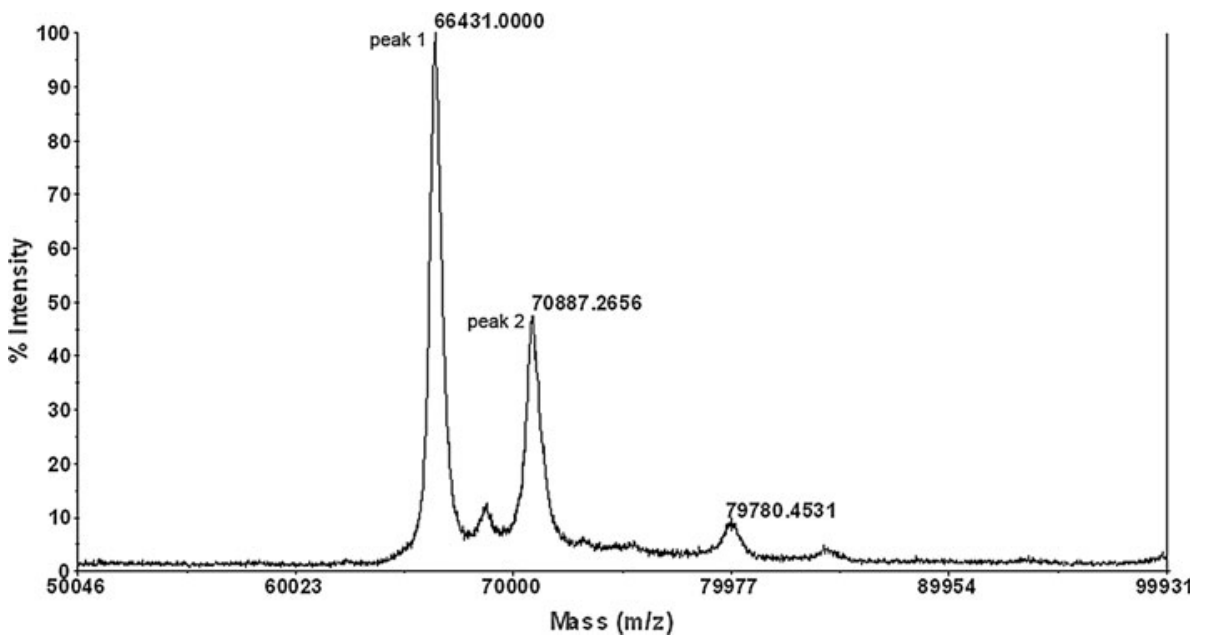

vacuolar proteases such as carboxypeptidase Y [11], thus disruptants GS115 pep4 $\triangle$ (proteinase A deficient) and GS115 pep $4 \triangle$ prbl $\triangle$ (proteinases A and B deficient) were also investigated. As shown in Fig. 7, fragment $d l$ was diminished significantly in strain GS115 pep $4 \triangle$, compared to wild-type strain GS115, but no change was observed for fragment $d 2$ (Fig. 7, lane 3,4). A similar expression pattern was found in GS115 pep $4 \triangle p r b 1 \triangle$ as in GS115 pep $4 \triangle$ (Fig. 7, lane 1, 3). These results suggest that proteinase A is involved in the formation of fragment $d l$ in a direct or indirect way, while proteinase B is less important. Besides, we found that the pep $4 \triangle$ disruptant seemed somewhat beneficial for reduction of $\sim 45 \mathrm{kDa}$ HSA-truncated fragment. This observation was more discernible in another albumin fusion protein HSA/IL1Ra (Fig. S2).

From the above results, we found that PEP4 disruption benefited the elimination of $d l$ fragment and YPSI disruption was beneficial for reduction of $d 2$ fragment. Thus, we further constructed a double disruptant GS115 pep $4 \triangle$ yps $1 \triangle$ and tested its effects for the production of HSA/PTH (1-34) fusion protein. The results showed that disruption of PEP4 and YPS1 genes yielded a significant increase in the proportion of intact HSA/PTH (1-34) by inhibition of degradation fragments $d 1$ and $d 2$, as compared to the wild-type host and single disruption of either PEP4 or YPS1 (Fig. 7, lane 2), with $80 \%$ of the secreted product remaining intact in pep4 $\triangle y p s 1 \triangle$ (Table 2$)$. The percentage of intact HSA/PTH (1-34) produced in other strains was about $47 \%$ for both pep $4 \triangle$ and pep $4 \triangle p r b 1 \triangle, 42 \%$ for yps $1 \triangle$, and $30 \%$ for GS115 wild-type host (Table 2).

Fed-batch fermentation of GS115 pep $4 \triangle y p s 1 \triangle$ mutant

A bioreactor culture was used to verify the shake flasks results under more controlled conditions. A fed-batch mode was applied and a cellular yield of $\sim 200 \mathrm{~g} / \mathrm{L}$ wet cells was achieved before methanol induction. Figure 8, for the pep4 $\triangle$ yps $1 \triangle$ mutant, most secreted protein remained intact during the $33 \mathrm{~h}$-fermentation, although degradation products gradually increased after $25 \mathrm{~h}$. However, for the wild-type strain, the intact HSA/PTH (1-34) was almost undetectable as early as after $16 \mathrm{~h}$ (and also after $30 \mathrm{~h}$; Fig. S4). This indicates that the pep4 $\triangle$ yps $1 \triangle$ mutant is advantageous over the wild-type by reducing the extent of proteolysis.

\section{Discussion}

The purpose of this study was to construct and develop an effective recombinant expression system in $P$. pastoris for high level production of human PTH (1-34) with HSA as a carrier protein in order to ultimately extend the biological half-life of native PTH (1-34). The fact that severe degradation occurred during $\mathrm{HSA} / \mathrm{PTH}$ (1-34) production not only resulted in a low yield of intact HSA/PTH (1-34), but also made it more difficult for downstream purification of intact recombinant protein. The proteolytic degradation is partly due to the high level of endogenous protease activity in the host cell, which is elicited by methanol induction causing stress on the cells [21]. Also, extracellular protease levels of $P$. pastoris tend to increase over time [21] and this seems to match our initial observation that the degradation of HSA/PTH (1-34) was dominant at later stage of cultivation.

In this research, our first attempt was to analyze the effect of each yapsin member on HSA/PTH (1-34) protein production by disruption of the individual genes, and the function of proteinases A and B were evaluated as well (Table 1). Although not sensitive enough to distinguish subtle differences of band patterns, PAGE analyses is a convenient visualized method for comparably evaluating the effects of these suspicious proteases on intact HSA/ PTH (1-34) production in this study. As we found that the 
Fig. 7 PAGE analysis of recombinant HSA/PTH (1-34) secreted through different protease-deficient strains. a Native PAGE gels of three independent batches of cultivation. For each strain, cells were induced in BMMY medium for $72 \mathrm{~h}$ and the equivalent of $20 \mu \mathrm{l}$ of clarified supernatant was loaded for all samples. b The relative abundance of intact protein (i) and cleaved protein ( $d 1$ and $d 2$ ) was determined by scanning with Quantity One Software version 4.5.0 (Bio-Rad), values were indicated as mean \pm S.D, from three independent batches of cultivation. Calculation of $\sim 45 \mathrm{kDa}$ fragment was not included since densitometry of $\sim 45 \mathrm{kDa}$ fragment at low optical density was unreliable
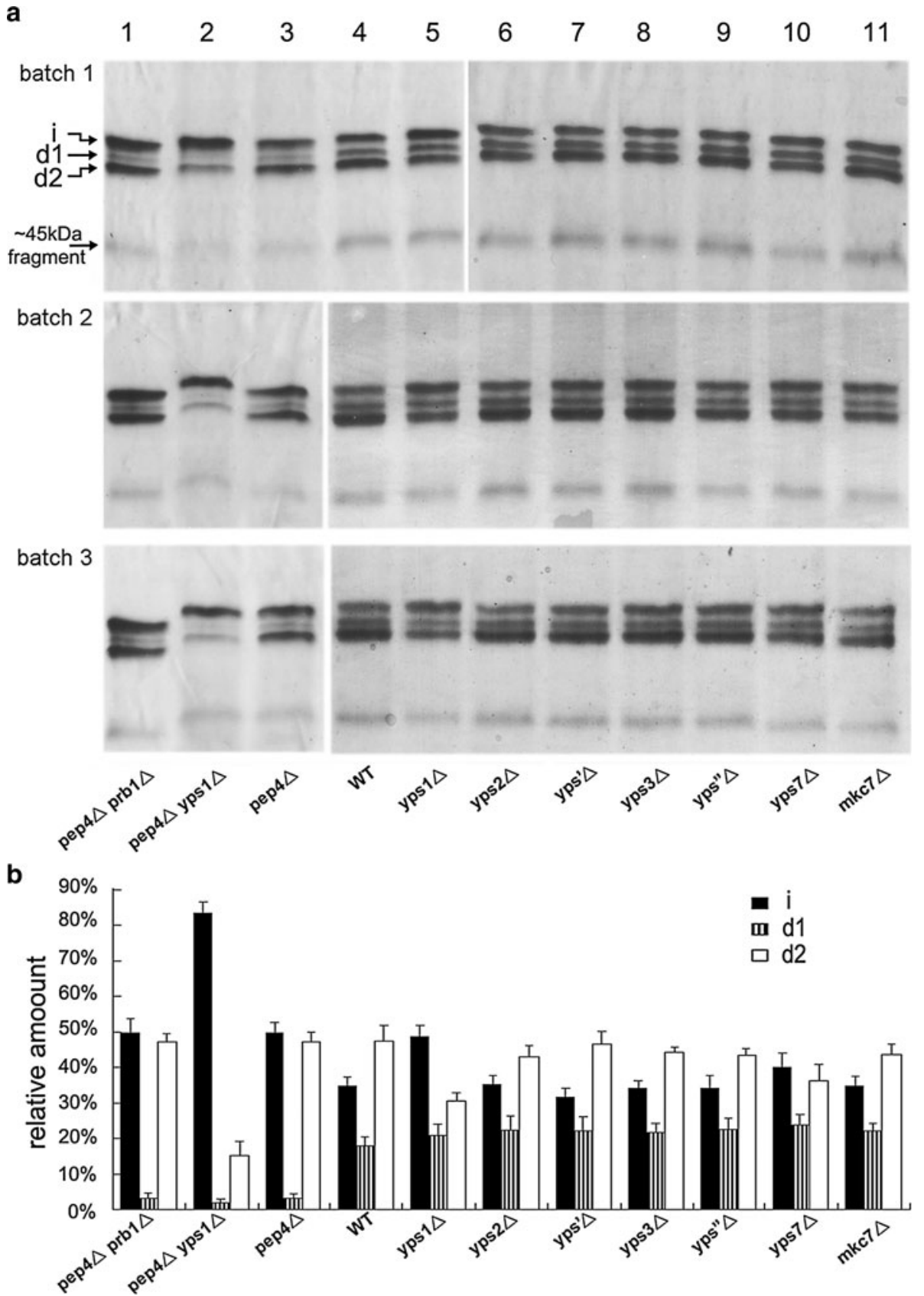

band patterns of the seven single-yapsin disruptants were quite similar to wild-type GS115 except for YPSI $\triangle$ disruptant, in order to make the densitometry of PAGE bands as accurate as possible, we compared the band patterns between 10 and $20 \mu \mathrm{l}$ of supernatants from GS115 strain to make sure that interference with density saturation was excluded (Fig. 9a). Besides, supernatants of these singleyapsin-disruptants from one batch were also loaded in $10 \mu \mathrm{l}$, and the result was similar as loaded in $20 \mu \mathrm{l}$ (Fig. 9b).

An important finding in this study is that the pep4 $\triangle$ disruptant can efficiently eliminate the prominent cleavage in PTH (17-34) portion (for degradation fragment $d 1$ ). Meanwhile, the proportion of intact HSA/PTH (1-34) was visibly improved by yps $1 \triangle$ disruptant, in which the prominent cleavage in PTH (1-17) sequence (for degradation fragment $d 2$ ) was considerably diminished, as compared with wild-type strain. Previous work reported that yapsin activity was almost undetectable in response to a yps $1 \triangle$ disruptant, suggesting that yapsin 1 might represent the major yapsin activity in $P$. pastoris [24]. This may partially explain why we did not observe the same positive effect in the other six single-yapsin-deficient strains as in yps $1 \triangle$ disruptant. Since the yps $1 \triangle$ disruptant alone is not 
Table 2 Yield of intact protein in wild type and PEP4/YPS1 disruptant strains

\begin{tabular}{ll}
\hline Strain & Yield of intact protein $(\%)^{\mathrm{a}}$ \\
\hline WT & $30 \pm 2$ \\
pep4 $\triangle$ & $47 \pm 3$ \\
pep4 $\triangle$ prb1 $\triangle$ & $47 \pm 3$ \\
yps1 $\triangle$ & $42 \pm 3$ \\
pep4 $\triangle$ yps1 $\triangle$ & $80 \pm 3$ \\
\hline
\end{tabular}

${ }^{\text {a }}$ Data are mean \pm S.D., acquired from three independent batches of cultivation. For each batch, supernatants of each strain were obtained after $3 \mathrm{~d}$-induction at $30^{\circ} \mathrm{C}$ and subjected to native PAGE analysis. Yields were quantified by scanning with Quantity One Software version 4.5.0 (Bio-Rad)

sufficient enough for total elimination of fragment $\mathrm{d} 2$, it is possible that all the yapsins synergistically participate in the degradation while activities of yapsin members vary widely. Also, all yapsins recognize basic residues and therefore the exact substrate specificity of the various yapsins and their involvement in the degradation of a particular protein may overlap [18, 19]. Cho et al. [5] also pointed out that though the yps $1 \triangle$ disruptant was advantageous over the wild-type strain, the effective prevention of the cleavage of secretory hPTH (1-84) in S. cerevisiae

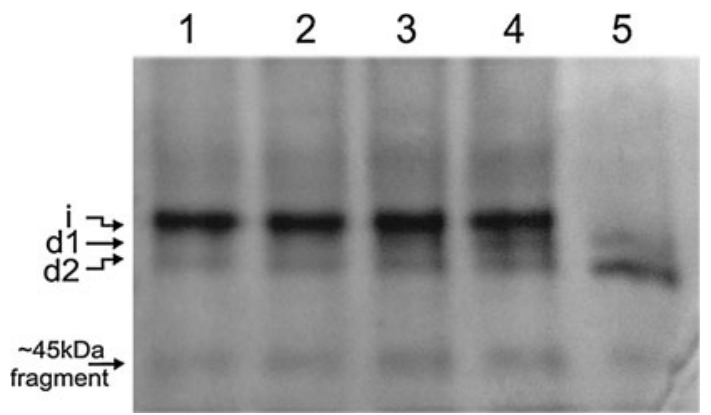

Fig. 8 Fed-batch fermentation comparison of GS115 pep4 $\triangle$ yps $1 \triangle$ and wild-type GS115 by native PAGE analysis. Lane 1-4 supernatants of GS115 pep4 $\triangle$ yps $1 \triangle$ after $21,25,29,33 \mathrm{~h}$ methanol induction; lane 5 supernatants of GS115 after $16 \mathrm{~h}$ methanol induction

Fig. 9 a Band patterns comparison between 10 and $20 \mu \mathrm{l}$ of supernatants from GS115 strain by native PAGE analysis. b Band patterns analysis of the seven singleyapsin-disruptants by loading $10 \mu \mathrm{l}$ of supernatants was only observed in multiple mutants containing at least the simultaneous disruption of YPS1, YPS2 and YPS3 genes. Thus, comprehensive analyses of various YPS disruption combinations in $P$. pastoris are necessary in further studies to evaluate whether there is an optical combination of multiple-yapsin disruptions for proteolytic inhibition. And also other yapsin-sensitive proteins should also be applied as recombinant protein models.

Yao et al. [26] have reported a minor reduction of the $\sim 45 \mathrm{kDa}$ fragment when they expressed an albumin fusion protein HSA/AX15 (R13 K) by a ypsl-disrupted P. pastoris strain, thus they speculated the existence of other YPS homologs in P. pastoris. However, in our study, none of the single-yapsin-deficient strains showed significant reduction of the $\sim 45 \mathrm{kDa}$ HSA-truncated fragment. This might partially be caused by the unreliable densitometry analysis (relative to background) of $\sim 45 \mathrm{kDa}$ fragment at low optical density. Thus, the densitometric calculations of the $\sim 45 \mathrm{kDa}$ fragment was excluded. But it is still possible that all the yapsins synergistically participate in the degradation. It is interesting for us to find that the pep4 $\triangle$ disruptant seemed somewhat beneficial for reduction of $\sim 45 \mathrm{kDa}$ HSA-truncated fragment (Fig. S2), suggesting the contribution of proteinases $A$ to the formation of $\sim 45 \mathrm{kDa}$ HSA-truncated fragment in $P$. pastoris.

Interestingly, Vad et al. [23] reported in their previous study that no increase in the absolute amount of intact PTH (1-84) was found using strain SMD1163 (GS115 pep $4 \triangle$ prb1 $\triangle$ ), whereas addition of EDTA to the medium could obtain higher hormone yield. Although the apparent differences between Vad's observations and ours seem unexplained, we could still speculate that PTH is sensitive to multiple proteases.

Finally, as mentioned above, pep $4 \triangle$ disruptant and yps $1 \triangle$ disruptant showed the most obvious effects on degradation reduction of HSA/PTH (1-34) fusion protein among the nine selected proteases in the first-round evaluation, thus a double disruptant (pep4 $\triangle$ yps $1 \triangle$ ) was constructed as the preferred attempt for multiple disruption. The pep $4 \triangle y p s 1 \triangle$ disruptant turned out to be a more effective host for minimizing proteolysis of HSA/PTH

\footnotetext{
10 $\mu$ l of supernatants
}

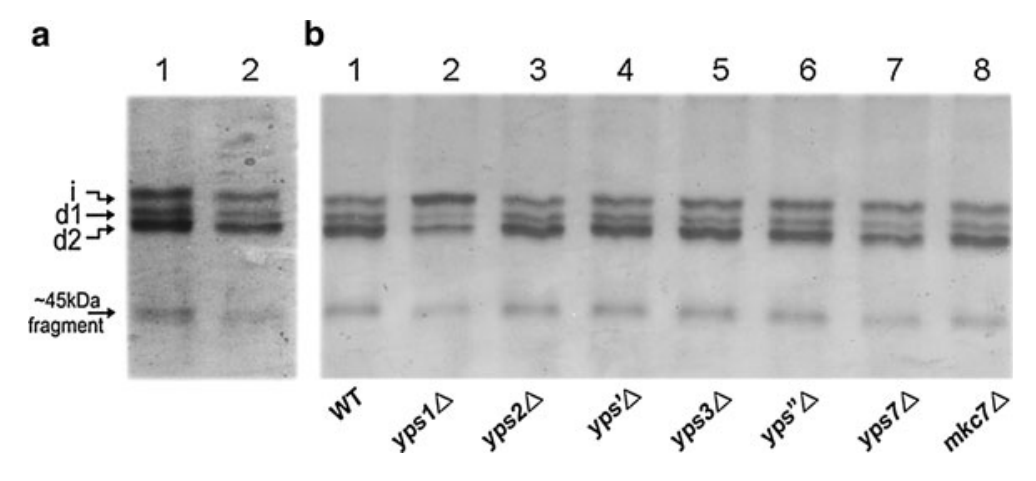


(1-34) and for its improved production by $P$. pastoris, as compared to wild-type or single disruption of either PEP4 or YPS1.

We have also tested the pep4 $\triangle$ yps $1 \triangle$ disruptant in fermentation mode. Although degradation products gradually increased after $25 \mathrm{~h}$ in pep $4 \triangle y p s 1 \triangle$ disruptant, the result indicates superiority of this double disruptant over wild-type strain. Of course, to minimize the loss of intact HSA/PTH (1-34), it is also necessary to optimize the fermentation process of pep $4 \triangle y p s 1 \triangle$ disruptant in further studies.

In the future, to develop a more efficient recombinant protein production system, further investigations are needed for genetic manipulation of $P$. pastoris as well as the optimization of the cultivation conditions. Besides, with the help of genomic sequence project of $P$. pastoris, not only the attempt of multiple-yapsin disruptions but also systematic and comprehensive analyses of a wider range of distinct proteases would be facilitated.

Acknowledgments This work was financially supported by two grants (No. 2007C03001-2 and No. 2010C13006) from Science and Technology of Department of Zhejiang Province, China. The authors would like to thank Professor David Wilson for his critical comments of this manuscript and helpful discussion.

Open Access This article is distributed under the terms of the Creative Commons Attribution License which permits any use, distribution, and reproduction in any medium, provided the original author(s) and the source are credited.

\section{References}

1. Bourbonnais Y, Larouche C, Tremblay GM (2000) Production of full-length human pre-elafin, an elastase specific inhibitor, from yeast requires the absence of a functional yapsin 1 (Yps1p) endoprotease. Protein Expr Purif 20(3):485-491. doi:10.1006/ prep. 2000.1338

2. Cawley NX, Chen HC, Beinfeld MC, Loh YP (1996) Specificity and kinetic studies on the cleavage of various prohormone monoand paired-basic residue sites by yeast aspartic protease 3. J Biol Chem 271(8):4168-4176. doi:10.1074/jbc.271.8.4168

3. Cawley NX, Wong M, Pu LP, Tam W, Loh YP (1995) Secretion of yeast aspartic protease 3 is regulated by its carboxy-terminal tail: characterization of secreted YAP3p. Biochemistry-Us 34(22):7430-7437. doi:10.1021/bi00022a016

4. Chen J, Sun HY, Yang Y, Wang XF, Chen SQ (2008) Construction, expression and characterization of recombinant fusion protein HSA-PTH (1-34) in Pichia pastoris. Zhejiang Da Xue Xue Bao Yi Xue Ban 37(2):126-133

5. Cho EY, Cheon SA, Kim H, Choo J, Lee DJ, Ryu HM, Rhee SK, Chung BH, Kim JY, Kang HA (2010) Multiple-yapsin-deficient mutant strains for high-level production of intact recombinant proteins in Saccharomyces cerevisiae. J Biotechnol 149(1-2): 1-7. doi:10.1016/j.jbiotec.2010.06.014

6. Copley KS, Alm SM, Schooley DA, Courchesne WE (1998) Expression, processing and secretion of a proteolytically- sensitive insect diuretic hormone by Saccharomyces cerevisiae requires the use of a yeast strain lacking genes encoding the Yap3 and $\mathrm{Mkc7}$ endoproteases found in the secretory pathway. Biochem J 330:1333-1340

7. De Schutter K, Lin YC, Tiels P, Van Hecke A, Glinka S, Weber-Lehmann J, Rouze P, de Peer YV, Callewaert N (2009) Genome sequence of the recombinant protein production host Pichia pastoris. Nat Biotechnol 27(6):561-566. doi:10.1038/ Nbt.1544

8. Guan B, Lei J, Su S, Chen F, Duan Z, Chen Y, Gong X, Li H, Jin J (2012) Absence of Yps7p, a putative glycosylphosphatidylinositol-linked aspartyl protease in Pichia pastoris, results in aberrant cell wall composition and increased osmotic stress resistance. FEMS Yeast Res 12(8):969-979. doi:10.1111/ 1567-1364.12002

9. Idiris A, Bi K, Tohda H, Kumagai H, Giga-Hama Y (2006) Construction of a protease-deficient strain set for the fission yeast Schizosaccharomyces pombe, useful for effective production of protease-sensitive heterologous proteins. Yeast 23(2):83-99. doi: 10.1002/yea.1342

10. Jin FJ, Watanabe T, Juvvadi PR, Maruyama J, Arioka M, Kitamoto K (2007) Double disruption of the proteinase genes, tppA and pepE, increases the production level of human lysozyme by Aspergillus oryzae. Appl Microbiol Biotechnol 76(5):1059-1068. doi:10.1007/s00253-007-1088-4

11. Jones EW (1991) Three proteolytic systems in the yeast saccharomyces cerevisiae. J Biol Chem 266(13):7963-7966

12. Kang HA, Rhee SK, Sohn MJ, Kim JY (2004) Hansenula porlymorpha yapsin deficient mutant strain and process for the preparation of recombinant proteins using the same. International Application Number: PCT/KR2003/001279

13. Kerry-Williams SM, Gilbert SC, Evans LR, Ballance DJ (1998) Disruption of the saccharomyces cerevisiae YAP3 gene reduces the proteolytic degradation of secreted recombinant human albumin. Yeast 14(2):161-169. doi:10.1002/(SICI)1097-0061 (19980130)14:2<161:AID-YEA208>3.0.CO;2-Y

14. Kobayashi K, Kuwae S, Ohya T, Ohda T, Ohyama M, Tomomitsu K (2000) High level secretion of recombinant human serum albumin by fed-batch fermentation of the methylotrophic yeast, Pichia pastoris, based on optimal methanol feeding strategy. J Biosci Bioeng 90(3):280-288. doi:10.1016/S1389-1723 (00)80082-1

15. Kuroda K, Kitagawa Y, Kobayashi K, Tsumura H, Komeda T, Mori E, Motoki K, Kataoka S, Chiba Y, Jigami Y (2007) Antibody expression in protease-deficient strains of the methylotrophic yeast Ogataea minuta. FEMS Yeast Res 7(8):1307-1316. doi:10.1111/j.1567-1364.2007.00291.x

16. Luck MD, Carter PH, Gardella TJ (1999) The (1-14) fragment of parathyroid hormone $(\mathrm{PTH})$ activates intact and amino-terminally truncated PTH-1 receptors. Mol Endocrinol 13(5):670-680. doi: 10.1210/me.13.5.670

17. Neer RM, Arnaud CD, Zanchetta JR, Prince R, Gaich GA, Reginster JY, Hodsman AB, Eriksen EF, Ish-Shalom S, Genant HK, Wang O, Mitlak BH (2001) Effect of parathyroid hormone (1-34) on fractures and bone mineral density in postmenopausal women with osteoporosis. N Engl J Med 344(19):1434-1441. doi:10.1056/NEJM200105103441904

18. Olsen V, Cawley NX, Brandt J, Egel-Mitani M, Loh YP (1999) Identification and characterization of Saccharomyces cerevisiae yapsin 3, a new member of the yapsin family of aspartic proteases encoded by the YPS3 gene. Biochem J 339:407-411. doi: 10.1042/0264-6021:3390407

19. Sievi E, Suntio T, Makarow M (2001) Proteolytic function of GPI-anchored plasma membrane protease Yps1p in the yeast vacuole and Golgi. Traffic 2(12):896-907. doi:10.1034/j.16000854.2001.21205.x 
20. Silva CI, Teles H, Moers AP, Eggink G, de Wolf FA, Werten MW (2011) Secreted production of collagen-inspired gel-forming polymers with high thermal stability in Pichia pastoris. Biotechnol Bioeng 108(11):2517-2525. doi:10.1002/bit.23228

21. Sinha J, Plantz BA, Inan M, Meagher MM (2005) Causes of proteolytic degradation of secreted recombinant proteins produced in methylotrophic yeast Pichia pastoris: case study with recombinant ovine interferon-tau. Biotechnol Bioeng 89(1):102112. doi:10.1002/Bit.20318

22. Subramanian GM, Fiscella M, Lamouse-Smith A, Zeuzem S, McHutchison JG (2007) Albinterferon alpha-2b: a genetic fusion protein for the treatment of chronic hepatitis C. Nat Biotechnol 25(12):1411-1419. doi:10.1038/nbt1364

23. Vad R, Nafstad E, Dahl LA, Gabrielsen OS (2005) Engineering of a Pichia pastoris expression system for secretion of high amounts of intact human parathyroid hormone. J Biotechnol 116(3):251-260. doi:10.1016/j.jbiotec.2004.12.004
24. Werten MWT, de Wolf FA (2005) Reduced proteolysis of secreted gelatin and Yps1-mediated alpha-factor leader processing in a Pichia pastoris kex2 disruptant. Appl Environ Microb 71(5):2310-2317. doi:10.1128/AEM.71.5.2310-2317.2005

25. Whitfield JF, Morley P (1995) Small bone-building fragments of parathyroid hormone: new therapeutic agents for osteoporosis. Trends Pharmacol Sci 16(11):382-386. doi:10.1016/S0165-6147 (00)89079-3

26. Yao XQ, Zhao HL, Xue C, Zhang W, Xiong XH, Wang ZW, Li XY, Liu ZM (2009) Degradation of HSA-AX15(R13 K) when expressed in Pichia pastoris can be reduced via the disruption of YPS1 gene in this yeast. J Biotechnol 139(2):131-136. doi: 10.1016/j.jbiotec.2008.09.006

27. Zhou XS, Zhang YX (2002) Decrease of proteolytic degradation of recombinant hirudin produced by Pichia pastoris by controlling the specific growth rate. Biotechnol Lett 24(17):1449-1453. doi:10.1023/A:1019831406141 